\title{
Administración educativa y la gestión de los conflictos estudiantiles
}

\author{
Educational Administration and Management Student's Conflict
}

Recibido 30 setiembre 2014 • Aceptado 28 noviembre 2014 • Corregido 08 diciembre 2014

\author{
Magaly Valverde Quirós ${ }^{1}$ \\ Ministerio de Educación Pública \\ San José, Costa Rica \\ magalyvalverde@gmail.com
}

\begin{abstract}
Resumen. En este artículo se analiza el abordaje de los conflictos estudiantiles que realiza la gestión de la educación en un centro educativo de educación secundaria del Ministerio de Educación Pública de Costa Rica - el Liceo de Paraíso, en la provincia de Cartago, a partir de una investigación de enfoque cualitativo, tipo fenomenológica, ejecutada durante el año 2014, con la participación de estudiantes, docentes guías, orientadoras y el director quienes suministraron información por medio de instrumentos como cuestionarios, entrevistas y grupos focales. El estudio permitió determinar algunos elementos importantes relacionados con el fenómeno de los conflictos estudiantiles en los centros educativos y el papel que se ejecuta desde la administración de la educación; entre estos aspectos se consideran los principales tipos de conflictos existentes, tanto verbales como físicos, las causas y consecuencias más relevantes a nivel del estudiantado y para la institución educativa, así como la falta de programas para la prevención de conflictos estudiantiles que se emplean en las organizaciones educativas y las estrategias aplicadas desde la administración de la educación para el abordaje de los conflictos estudiantiles.
\end{abstract}

Palabras clave. Educación; administración educativa; conflictos; conflictos estudiantiles.

Abstract: This article analyzes the resolution of student conflicts in a public high school of the Costa Rican Ministry of Education - the Liceo de Paríso in the province of Cartago. The focus lies on how the administrative staff managed specific scenarios through a qualitative, phenomenological, investigational approach implemented at the school throughout 2014. Data is based on questionnaires, interviews and focus groups collected among the students, guidance counselors, the high school principal and staff. The research aims at determining the elements that characterize struggles among students at high schools - mainly verbal and physical conflicts. The paper looks at the most relevant causes and consequences for both the institution and the students, lack of programs to prevent such occurrences and daily planning by school administrators to counteract such encounters as well as raise awareness among the high school community.

1 Máster en Administración Educativa de la Universidad de Costa Rica (UCR). Licenciada en Ciencias de la Educación con énfasis en Orientación de la Universidad de Costa Rica (UCR). Bachiller en Ciencias de la Educación con énfasis en Orientación de la Universidad de Costa Rica (UCR). Actualmente es Orientadora del Liceo Laboratorio Emma Gamboa, de la Universidad de Costa Rica en Moravia, del Ministerio de Educación Pública (MEP). 
Key Words: education; administrative staff; conflicts; student conflicts; emotional education and alternative conflict resolution.

\section{Introducción}

El mundo ha experimentado una serie de cambios en diversos campos del saber, producto de una secuencia transformadora, que la misma era de la globalización ha sido testigo y protagonista.

Ante esto, la vida en un mundo globalizado ha posibilitado que el ser humano tenga más acceso a una serie de información y formación, desde donde el papel de la sociedad, medios de comunicación e información han tenido que trascender grandemente, en miras de acoplarse a esa serie de innovaciones que la vida actual ha generado en las personas. De ahí, que teóricos como Torres (2004) argumentan que la globalización ha influenciado sectores como la educación, principalmente a nivel latinoamericano, en la medida que le ha impactado en tres focos de interés: la economía para el financiamiento de la educación, la relación entre educación y trabajo, la creación de estándares de excelencia académica internacional.

Lo que implica en alguna medida, visualizar la globalización y su impacto en el país, propiamente en el sector educación, desde una serie de cambios suscitados, no solo para efectos de políticas educativas sino en diversidad de proyectos a nivel nacional e institucional; así como un cambio en las funciones reales que se le asignan a los centros educativos como entes formadores de las sociedades actuales, desde las acciones a realizarse con la niñez y juventud que asiste, día a día, a los centros educativos.

Lo anterior conlleva, según Arroyo (2009), una educación que desde las instituciones educativas debe "difundir los saberes, valores e ideas dominantes que pretenden modelar la sociedad, por medio de la modelación de la conducta y de la conciencia de los individuos que la componen" (p. 22). De manera que las organizaciones educativas como formadoras, deben luchar contra una serie de fenómenos que aquejan a la sociedad actual para, en alguna medida, contrarrestar la influencia negativa que estas puedan suscitar en la vida de las poblaciones estudiantiles actuales; tal es el caso de los actos de violencia y de conflictos que tan a menudo ocurren entre las poblaciones estudiantiles en las instituciones educativas, a lo largo y ancho del territorio nacional.

Considerando la realidad educativa actual que configura un importante reto para la administración de la educación, Garbanzo y Orozco (2007) explican que esta disciplina es responsable de conducir, de forma óptima, los procesos de gestión educativa en las diferentes esferas de la educación. Razón que conlleva a que, como administradores de la educación, se deba favorecer un modelo de educación curricular afín con la sociedad actual, sus necesidades y expectativas, y a desarrollarse en cada una de las instituciones educativas, según condiciones contextuales, lo que implicará acciones y transformaciones desde los diseños y estrategias 
curriculares hasta políticas integrales, en procura del bienestar de la población estudiantil como seres humanos y estudiantes en formación.

De modo, que desde la administración de la educación como la responsable y protagonista de las transformaciones y cambios escolares en todas las dimensiones del quehacer educativo, se deben tomar en cuenta diversos aspectos, tanto internos, propios de cada institución, como externos, según las condiciones sociales, económicas y políticas que rodean a la institución, con la finalidad de realizar una gestión acorde con las condiciones y necesidades propias de las poblaciones estudiantiles que se atienden en los diferentes centros educativos del país.

Con base en las consideraciones anteriores, se plantea la necesidad de desarrollar en las instituciones educativas, espacios y estrategias para abordar la temática de los conflictos estudiantiles, no solo haciendo frente al problema como tal sino en el que se desarrollen mecanismos para prevenirlos $y$, de esta manera, desde los centros educativos contribuirse en la formación integral de sus poblaciones estudiantiles, con el fin de favorecer un valioso aporte en materia de formación curricular y conductual, moral y social. Para cumplir con estas necesidades educativas de las organizaciones educativas, se requiere de un liderazgo pedagógico para asumir y resolver, de manera asertiva, los conflictos estudiantiles.

De esta forma, el estudio determina como objetivos generales y específicos:

\section{Objetivo general:}

- Analizar el abordaje que se realiza desde la gestión de la educación, en el manejo de conflictos estudiantiles en el Liceo de Paraíso.

\section{Objetivos específicos:}

- Determinar los principales tipos de conflictos estudiantiles, que se generan entre la población estudiantil del Liceo de Paraíso.

- Reconocer las principales causas asociadas a conflictos estudiantiles a nivel personal, institucional y familiar, en el Liceo de Paraíso.

- Reconocer las principales consecuencias asociadas a conflictos estudiantiles a nivel personal, institucional y familiar, en el Liceo de Paraíso.

- Determinar los diferentes procesos, que se desarrollan desde la gestión de la educación, en la prevención de conflictos estudiantiles en el Liceo de Paraíso.

- Identificar los diferentes procesos, que se desarrollan desde la gestión de la educación, en el abordaje de conflictos estudiantiles en el Liceo de Paraíso. 


\section{Fundamentación teórica}

\section{Conceptualización de la educación}

La educación desde sus inicios, ha tenido esa función formadora del ser humano; conforme han evolucionado sus fines, se modifica, pero siempre guardando su objetivo. Rodríguez (2006) considera que, desde siempre, la escuela ha tenido dos responsabilidades fundamentales: una instructiva y otra formativa. Una destinada al desarrollo intelectual y la otra, a la formación personalsocial, ya que las personas más que seres humanos con capacidades, habilidades e intereses, son seres que requieren que esas cualidades sean desarrolladas y explotadas y es ahí, donde la educación irrumpe, marcando un valioso espacio en la vida del ser humano, como ser personal y social.

Una educación como tal, trasciende de lo individual a lo colectivo, pues permite a las personas desarrollarse desde procesos de aprendizaje y enseñanza aptos para sus expectativas, necesidades y contexto. Para Dengo (2011, p. 6), es esa educación que"se dirige a los niños, a los adolescentes, a los jóvenes y los adultos, de acuerdo con sus respectivas necesidades y edades", lo que implica una contextualización apropiada de los procesos educativos, en función de las poblaciones estudiantiles a los que van dirigidos, donde se les forme no solo en elementos intelectuales para la vida, sino en seres morales y sociales.

Condición que se podría sintetizar en una de las frases del pedagogo brasileño Freire, "Los hombres se educan entre sí; ; de esta forma, el proceso educativo se concibe como aquel que se fundamenta y se sustenta en la sociedad, puesto que la educación no edifica al ser humano en abstracto, sino que requiere de una estructura social para su desarrollo. De ahí, esa visión social de la educación que implica la formación para la vida, desde el desarrollo de herramientas para interactuar socialmente, en un clima social cada día más cambiante, donde las problemáticas, eventos y situaciones propias de país, determinan en alguna medida el curso que debe tomar la educación, como garante de la formación de generación en generación. Punto en el que radica la importancia de abordar la temática de los conflictos estudiantiles ocurridos en la instituciones educativas, como mecanismo para incluir esa formación social en el proceso educativo que se facilita en los diferentes centros educativos de Costa Rica.

Ante esto, las instituciones educativas, bajo la dirección de profesionales en administración de la educación, deben tomar las medidas respectivas para dirigir cada centro educativo en función de estos principios; lo anterior, en beneficio de las poblaciones educativas al recibir los procesos educativos gestados y de las mismas comunidades y sociedad, en general, por ese papel socializador que desde la educación, se supone realizar y desarrollar.

\section{Administración de la educación}

La administración, como disciplina, es una herramienta de suma importancia dentro de las organizaciones, la cual considerando a Jiménez (2000), citado por López (2012): 
(...) ha de ser un instrumento dinámico, capaz de convertir la organización en una actividad, concebida como un sistema de vasos comunicantes, y que la oficina central sea el órgano de servicio en donde se coordinen y se unifiquen las diferentes labores de toda una comunidad educativa internacional. (p. 28)

Aspecto que implica que la administración realiza tanto las coordinaciones respectivas para el logro de las metas propuestas a nivel organizacional, como facilitar el desarrollo de procesos, en virtud de las necesidades y expectativas de la institución que lidera. La administración de la educación debe plantear su accionar mediante un proceso reflexivo y consensuado a la luz de la planificación educativa, mediante planes, programas y proyectos.

De modo que, desde la administración, es prioritario tomar decisiones para guiar por buen camino los ideales y objetivos de la organización, así como el asumir esa jefatura, desde una visión de liderazgo, delimitando funciones y distribuciones del personal que tenga a su cargo.

De esta manera, se concibe la administración de la educación como aquella disciplina que se desarrolla en los centros educativos y que, según Garbanzo y Orozco (2007), tiene como principal reto la construcción de un desarrollo sostenible desde la equidad, calidad y justicia, en cada una de las dimensiones que esta disciplina le corresponde. De modo, que es función de la administración de la educación, que en los centros educativos se brinde no solo un proceso de enseñanza y aprendizaje de calidad, sino que esté acorde con las necesidades de la población a las que se dirige; es decir, desde el propio contexto en donde se desarrolla ese centro educativo.

De esta forma, las personas profesionales en el campo de la administración de la educación han de ser facilitadoras de procesos educativos acordes con las necesidades, expectativas y realidades de los centros educativos que dirijan; sobre estos profesionales recae el compromiso y la responsabilidad de dirigir los pasos y hacer las gestiones pertinentes para que el proceso educativo sea exitoso y pertinente a los retos sociales, culturales y humanos actuales.

Como disciplina, la administración de la educación se debe a un modelo de sociedad y se desarrolla, principalmente, en cada una de las instituciones educativas; es concebida por Garbanzo y Orozco (2007) como aquella responsable de conducir, de forma óptima, los procesos de gestión educativa en las diferentes esferas de la educación. La administración de la educación debe orientar y guiar su accionar respondiendo a un modelo de sociedad, el cual se instituye en la Constitución Política, la Ley Fundamental de Educación y la Política Educativa nacional; es decir, un marco jurídico que respalda su quehacer diario.

Entre los fines primordiales de la administración de la educación, desde el accionar del gestor de la educación, debe contemplarse el ofrecer la educación como un servicio, en favor de la sociedad, garante de desarrollo humano, formación integral de las poblaciones estudiantiles y ante todo, promotora de procesos de socialización oportunos y acordes con las realidades del momento. Al respecto, López (2012) considera que la persona administradora debe emplear cada uno de los insumos que tenga a su disposición, para orientar de la mejor manera sus funciones: 
La administración de la educación proporciona al gestor de la educación un instrumento que le ayude a estudiar la organización y la estructura de las instituciones, que permitirá orientar en las funciones de planificación, organización, coordinación, dirección y control en el programa a seguir. (p. 45)

Funciones, desde la administración de la educación, que permitirán a este profesional no solo dirigir un centro educativo, sino llevarlo por un camino óptimo a futuro, desde una planificación, coordinación y dirección oportuna ante las necesidades y requerimientos propios de la población y de la organización educativa.

Para tales fines, la administración de la educación le posibilita a su gestor la toma de decisiones adecuadas y pertinentes ante las múltiples necesidades institucionales y de las poblaciones que se atienden. Lo que es retomado por Vargas (2008), al mencionar que "la administración educativa supone una deliberación y una toma de decisiones responsable, que permita a los profesores de la escuela asumir un papel activo en la construcción de una comunidad educada y capaz de establecer un orden dinámico y democrático" (p. 3). Una toma de decisiones que conlleva un actuar responsable desde la dirección del centro educativo, para una consecuente coordinación, conducción y actuación en función de los procesos educativos, que dirija los destinos de las poblaciones estudiantiles que ahí estudian. Es necesario que los profesionales en esta disciplina, desarrollen habilidades y herramientas que les permitan lograr un mayor acercamiento con las poblaciones estudiantiles, con el fin de tomar decisiones y desarrollar acciones desde las necesidades, inquietudes y expectativas estudiantiles. Difícilmente, desde el desconocimiento y lejanía para con las poblaciones estudiantes, un administrador educativo va a conocer el tipo de estudiante y necesidades contextuales del centro educativo que lidera.

Asimismo, el desafío más complejo de la administración de la educación como disciplina inmersa en el sistema educativo, es concebir y propiciar la creación de un nuevo modo de conducir el funcionamiento de este, en donde el eje central sea el desarrollo de capacidades humanas, técnicas e institucionales para llevar a cabo las nuevas tareas en un contexto social diferente y cambiante (Vargas, 2008). Consideraciones que conllevan una labor integral y proactiva del gestor de la educación, desde donde se puntualicen los fines y objetivos institucionales y con ello, se marquen las expectativas y el curso a seguir en el presente, con miras a un futuro mejor. Además, la gestión escolar debe potenciar y estimular a los integrantes de la comunidad educativa un sentido de pertenencia hacia la institución, lo que favorecerá la consecución del Proyecto Operativo de Centro.

Una misión de la administración de la educación que, desde un abordaje integral de las necesidades del centro educativo, implica un actuar responsable y oportuno a las condiciones organizacionales en donde se labore; la temática de los conflictos estudiantes puede ser considerada como una de esas necesidades para ser tratada desde la gestión de la educación que le competa. 


\section{Gestión en las organizaciones educativas}

Las organizaciones educativas, como instituciones dirigidas por profesionales en administración de la educación, requieren de procesos de gestión acordes con las condiciones organizacionales propias que implican elementos que van desde la planificación, coordinación y dirección responsable, desde el gestor de la educación.

Ante ello, la gestión educativa en las organizaciones debe verse como un insumo y herramienta de suma utilidad desde la administración de la educación, la cual ejerce esa función de liderazgo y muestra habilidades para la toma de decisiones, con el fin de coordinar y llevar por una adecuado rumbo los objetivos organizacionales, tomando en cuenta cada una de las oportunidades, fortalezas y amenazas institucionales.

La gestión estratégica de las organizaciones constituye todos aquellos espacios de tiempo definidos (periodos) por el director, para crear y formar (concebir) las estrategias dirigidas a prevenir y organizar (preparar) en conjunto de conocimientos, cualidades, experiencias y habilidades que, individual y colectivamente, posee su personal (potencial), para hacerlas pasar una serie de estados, cada uno más perfecto que el anterior (desarrollar) y darles la fuerza necesaria (fortalecer) para alcanzar su razón de ser (misión) y traducir en realidad su imagen mental futura (visión). (Arroyo, 2009, p. 3)

De modo, que es evidente la diversidad de elementos que se confluyen cuando de gestión estratégica de las organizaciones educativas se habla; de ahí, lo valioso de que como gestores se demuestren todas aquellas competencias, habilidades y capacidades necesarias para dirigir los pasos y la dirección oportuna del centro educativo en donde se labore.

Una gestión estratégica de la educación permite que, a través de diversos procesos, se lleve a buen destino a las instituciones educativas del país, desde la puesta en práctica de todas aquellas acciones pertinentes al caso, las cuales son resumidas por Botero (2008) como aquel "conjunto de procesos, toma de decisiones y realización de acciones que permitan llevar a cabo las prácticas pedagógicas, su ejecución y evaluación" (p. 2). Tareas y estrategias a realizar que, de una u otra manera, van a marcar el camino a seguir por la organización educativa, tanto por la población estudiantil como por el personal que labora en ella, así como todas aquellas fuerzas vivas y agentes de la comunidad que intervienen en el devenir de la organización escolar; todos y cada uno de ellos y ellas, bajo la dirección de ese gestor de la educación, quien actúa como el capitán que lleva ese gran barco, denominado centro educativo.

Sin dejar de lado que, como gestores de la educación, se deben contemplar no solo los sucesos que ocurren a lo interior de las instituciones educativas, sino mirar muy de cerca los eventos que suceden a lo externo de estas, desde la misma realidad social, cultural y económica que, definitivamente, marcan ese momento y contexto imperante; este debe ser validado y tomado en cuenta a la hora de tomar decisiones desde la administración del centro educativo, 
en relación con los procesos de formación a ejecutarse con las poblaciones estudiantiles, desde sus necesidades y expectativas.

Se rescata, de esta manera, la temática del abordaje de conflictos estudiantiles como uno de esos elementos claves que se deben considerar desde la gestión de los centros educativos, por la masiva amenaza e influencia que este tipo de sucesos y eventos llenos de violencia han generado en el país, principalmente en las poblaciones más jóvenes, aquellas que son las más vulnerables de verse afectadas.

\section{Conflictos, fundamentos conceptuales}

El conflicto es conceptualizado, por autores como Cascón (2006, p. 24), como un proceso "inherente a las relaciones humanas. En el momento que varias personas estamos interaccionando en un mismo espacio por un determinado tiempo, van a surgir conflictos", lo que implica que las relaciones humanas, por el simple hecho de desarrollarse, van a concebirse como espacios en los que pueden surgir conflictos, dada la confluencia de diferencias que cada ser humano posee y manifiesta al relacionarse con sus semejantes.

Lo anterior se considera un punto clave en la medida que, al vivirse en sociedad, las personas no están ajenas a lo que sucede a su alrededor, no pueden pasar desapercibidas y mucho menos pueden obviar el relacionarse con otras personas, sea porque ocupen de sí o a su vez se requiera de los demás para la satisfacción de necesidades. De manera que, como seres humanos en constante relación con los otros, no se puede pasar ajeno al surgimiento de conflictos, pues van a ser parte del desarrollo de las mismas.

Muy unido a esta idea, Chacón (2012, p. 15) apunta a que "los conflictos forman parte de la cotidianidad del ser humano, es decir en la multiciplicidad de intereses, motivaciones y relaciones, el conflicto aparece como un componente natural, sustancial e ineludible". Razón de peso para valorar el conflicto como un elemento inherente a la vida de las personas, en la medida de que estas viven en constantes interacciones con las demás personas a su alrededor.

Sin obviar, que al converger una serie de particularidades propias de cada persona, esas interacciones van a desarrollarse en medio de algunas diferencias que van a marcar no solo el rumbo de estas, sino el surgimiento de posibles conflictos ante la exposición o debate de las múltiples discrepancias propias del ser humano y su entorno, que suelen suceder en el seno de la organización educativa, producto de los fenómenos psicosociales del contexto sociocultural al que pertenecen las personas. De ahí, que la administración de la educación debe asumir ese rol protagónico ante estos sucesos, como la encargada de enfrentar y resolver estas divergencias de la mejor manera posible.

De forma que el conflicto no está alejado de la naturaleza humana, sino que por el contrario, es parte de la vida misma. Al respecto, Rodríguez (2008) menciona que "existe un 
conflicto cuando aparecen actividades contrapuestas, cuando las acciones de una persona impiden u obstaculizan las acciones de otra que también intenta alcanzar sus metas" (p. 3). Lo anterior, implica que precisamente esas particularidades de cada quien, que al relacionarse con los otros se convierten en diferencias sean, en alguna medida, las causantes de conflictos en las interacciones humanas.

\section{Conflictos estudiantiles}

A nivel de centros educativos, principalmente si del Ministerio de Educación Pública se trata, existe el Reglamento de Evaluación de los Aprendizajes, el cual especifica una serie de deberes y derechos, así como directrices que la población estudiantil debe cumplir, con miras a lograr una adecuada convivencia. De igual forma, en el año 2012, el Ministerio de Educación Pública emitió una serie de protocolos para atender las dificultades y problemáticas que se presentan en los centros educativos a nivel estudiantil, como es el caso de situaciones de matonismo, bullying, portación de armas, entre otras.

Esta legislación actual permite a los centros educativos no solo tener los insumos para enfrentar situaciones problemáticas, sino contextualizar más el fenómeno de los conflictos estudiantiles como una problemática real y recurrente que ocurre a diario en las instituciones educativas.

Desde esta perspectiva, los conflictos estudiantiles se entienden como aquellos que se desarrollan a partir de las interacciones que se dan entre las poblaciones estudiantiles. Posición desde la que López (2012, p. 50) menciona que: “los conflictos estudiantiles, serán de provecho, siempre y cuando se le dé una adecuada intervención, ya que de los errores se aprende ( ) es decir la clave está en cómo tratar el conflicto, pero no caer en extremos y so se produzca violencia".

Lo que implica, que si bien los conflictos estudiantiles no se pueden evitar puesto que surgen en las relaciones entre estudiantes, si es posible que, a partir de una oportuna intervención institucional, se aprenda de ellos, evitando muchas de las diversas manifestaciones de violencia que se desencadenan, día con día, en los centros educativos del país.

De manera, que los conflictos entre los estudiantes pueden evitarse dentro de la aulas y en diferentes espacios de los centros educativos, debido a la multiplicidad de elementos que entran en juego a la hora de conformar y desarrollar sus relaciones interpersonales; lo importante del caso no es luchar contra su eliminación, sino que esos esfuerzos que se realicen en ese sentido, se dirijan hacia el cómo abordarlos y resolverlos.

Los conflictos son un fenómeno natural de todas las organizaciones. Por tanto, es normal que en las aulas y en los centros educativos se produzcan de forma continua. Así pues, debemos fijarnos no ya en la existencia de conflictos, sino en la forma de resolverlos en las aulas y en los centros. (Viñas, 2011, p. 13) 
El acontecer diario dentro de las instituciones educativas, son espacios totalmente propensos a que ocurran conflictos entre los estudiantes, tanto por diferencias entre ellos como por la misma ausencia de habilidades de comunicación y de relación interpersonal en las diferentes acciones que el quehacer educativo les implica. De ahí, que como gestores educativos se deben propiciar espacios para el abordaje de estas temáticas, no solo a nivel de intervención propiamente, sino desde la prevención de estas situaciones; en estas acciones pueden participar los diferentes entes educativos, con el fin de educar al estudiantado en habilidades sociales tan valiosas como la misma comunicación amerita.

Un conflicto no se debe confundir con violencia; si bien se relacionan ampliamente entre sí, se dan y llegan a procesos diferentes. Con respeto a esta diferenciación de términos, Esteve y Jordan (2001), citados por López (2012), argumentan que:

( ) no es legítimo asociar conflicto con violencia porque, mientras el conflicto responde a situaciones cotidianas de la vida social y escolar, en la que se dan enfrentamientos de intereses, discusión y necesidad de abordar el problema, la violencia es una de las maneras de enfrentarse a esta situación. (p. 82)

El conflicto entre estudiantes es la existencia de enfrentamientos y desacuerdos entre estos, pero la violencia va más allá, como una posible manifestación de un conflicto que, a raíz de un inadecuado manejo del mismo lleva al uso de la fuerza física, verbal y psicológica para resolverse.

De modo, que los conflictos estudiantiles se producen constantemente de manera que lo que determina que sean destructivos o constructivos no es su existencia, ya que siempre van a surgir, sino el modo en que sean manejados (Rodríguez, 2008). Un punto y accionar determinante que puede no solo marcar las pautas en correspondencia con las relaciones interpersonales a desarrollar, sino hasta el propio futuro y permanencia de las poblaciones estudiantiles dentro de los centros educativos; ya que en la medida que se dé un adecuado abordaje de los conflictos, más se fortalece el desarrollo interpersonal de estas poblaciones.

De ahí, el trascendental papel de las gestiones educativas de los centros educativo en el abordaje oportuno de esta temática tan común, entre las poblaciones estudiantiles, desde un accionar que permita la aplicación rigurosa de las directrices establecidas para tales fines, el compromiso y responsabilidad que se requieren, e incluso desde una visión humanista e integral, en beneficio de las poblaciones estudiantiles que se atienden. El administrador de la educación debe posicionar su rol, ejercerlo y tomar las decisiones del caso para promover espacios educativos desde una sana convivencia, en donde la comunidad educativa trabaje en miras de una mejora continua.

\section{Tipos de conflictos estudiantiles, que se presentan en las instituciones educativas}

Los conflictos se pueden dar en cualquier relación que se desarrolle entre personas, sea cual sea el ambiente en donde se desenvuelvan. De ahí, que existen diferentes tipos de 
conflictos, según la situación o eventos que los generen. Ante ello, autores como Ortega (1996), citado por López (2012), realiza la siguiente división de los conflictos en cinco tipos:

- Los conflictos de relación: se deben a fuertes emociones negativas, ligadas a una falsa y escaza comunicación.

- Los conflictos de información: se dan por la falta de información con que cuenta la persona para tomar decisiones correctas.

- Los conflictos de intereses: se originan por la competitividad entre las necesidades de las personas, desde donde se satisfacen las necesidades propias, dejando de lado y sacrificando las necesidades del otro.

- Los conflictos estructurales: se deben a estructuras muy competitivas, en donde se promueve no solo la competitividad sino también la opresión y condiciones desfavorables para quienes ahí se encuentren, tanto de manera individual como colectivamente.

- Los conflictos de valores: originados por sistemas de creencias y valores, considerados por las personas como incompatibles entre ellos.

Diversas formas de agrupar los conflictos, las cuales permiten ver el conflicto como algo mas multidimensional en la medida que entran en juego una serie de variables, que van desde aspectos concernientes a uso inadecuado de canales de comunicación y falta de información hasta condiciones propias de la formación de la persona, vinculadas con sus intereses, valores y creencias. Lo anterior, manifiesta no solo la complejidad del ser humano, sino diversos elementos que entran el juego cuando de relaciones humanas se trata; de ahí, que los conflictos se presenten con la constancia que lo hacen, sobre todo si se dan entre poblaciones de adolescentes quienes, ante sus múltiples cambios, son más complejos y cambiantes.

En esa etapa de la vida, los conflictos pueden ocasionarse por diversidad de circunstancias, por lo que se ubican en uno u otro tipo, según las implicaciones que se confluyan en esas relaciones; ante estas situaciones, las personas implicadas pueden decidir y dar un curso determinado a ese conflicto. Como gestores educativos se tiene el deber de formar a las poblaciones estudiantiles para darle un adecuado abordaje a los conflictos, con el fin que los centros educativos, más que promotores de violencia, sean espacios de crecimiento y superación personal e institucional.

Lo anterior implica un importante reto para el administrador de la educación, quien no solo debe buscar los medios para conocer y diagnosticar las problemáticas institucionales, como por ejemplo los conflictos estudiantiles, sino que debe emplear esta información como insumo para buscar, crear e implementar nuevas policitas y proyectos que permitan, al centro educativo como tal y a los propios estudiantes, buscar soluciones y mejorar los niveles de convivencia. 


\section{Causas de los conflictos estudiantiles en las instituciones educativas}

Al visualizar los conflictos como un proceso que se da y surge dentro de las relaciones interpersonales, y que están relacionados con todos aquellos eventos y elementos que a nivel contextual se desencadenan, el ser humano está expuesto a una serie de situaciones que, de una u otra forma, influyen en su conducta y por ende, en el surgimiento de conflictos.

Desde esta perspectiva, los conflictos están ligados a una serie de elementos como causas que, según Fernández (2007) podrían originar condiciones conflictivas en las relaciones interpersonales. Entre esas causas o agentes institucionales asociados al origen de los conflictos, este autor menciona:

- Agentes exógenos: se consideran todos aquellos elementos concernientes a la estructura social y que son externos al centro educativo. Entre estos agentes se pueden encontrar los agentes propios de la sociedad, medios de comunicación y la familia.

- Agentes endógenos: se incluyen todos aquellos aspectos que ocurren a lo interno del centro educativo, que van desde elementos propios de las relaciones interpersonales entre estudiantes, estudiantes-docentes, docentes-docentes y factores propios de la institución educativa en cuanto a sus disposiciones, reglamentos, organización y fines institucionales.

Entre los agentes exógenos que propician situaciones de conflicto entre estudiantes, condicionados al momento contextual e inclusive histórico de la realidad actual, se encuentran las diferentes problemáticas sociales, culturales y económicas por las que atraviesa el país, en donde aspectos como la inseguridad y la violencia son el punto principal de las calles y de los noticieros día a día. Esta realidad social se refleja en los agentes endógenos, propios del clima institucional del centro educativo que, aunque es un espacio más reducido en relación con la sociedad en general, de una u otra manera, es reflejo del acontecer nacional.

De modo, que tanto los factores externos como internos tienen un decisivo papel en la formación de las conductas del ser humano, y la forma de enfrentar diversas situaciones conflictivas que puedan surgir, como parte de las relaciones con los otros que se puedan desarrollar. Lo que implica todo un reto para las instituciones educativas, en materia de abordaje de conflictos, por lo que los esfuerzos deben dirigirse hacia las causas que puedan dar origen a estos, con la finalidad de desarrollar acciones para intervenir y comprender los factores originarios antes de que se conviertan propiamente en conflictos y mucho menos, permitir que se llegue a conductas o espacios de violencia.

\section{Consecuencias de los conflictos estudiantiles}

Los conflictos estudiantiles son un fenómeno que cada día se da con mayor frecuencia en los centros educativos, debido a multiplicidad de factores y razones que llevan a la población estudiantil a visualizar el conflicto como esa formar de resolver sus diferencias. 
Al darse un conflicto entre estudiantes, una serie de acciones o consecuencias se desencadenan, como medida para enfrentar lo sucedido y atender a la población estudiantil implicada. No obstante, muchas de esas acciones equivalen a elementos negativos, las cuales afectan tanto al centro educativo como a cada estudiante que se enfrenta a algún tipo de conflicto. Al respecto, Chacón (2012) hace referencia a esas posibles consecuencias que se pueden generar a partir de un conflicto:

(...) en las organizaciones educativas coexisten distintos tipos de conflictos; sus consecuencias habitualmente son de índole negativa como por ejemplo la disrupción, los problemas de disciplina (conflictos entre profesores (as) y alumnos (as), abuso de poder, falta de comunicación o ausencia total, maltrato físico y psicológico entre compañeros (bullying), vandalismo y daños materiales, violencia física (agresiones y extorsiones), y el acoso sexual. (p. 22)

Diferentes acciones van a afectar no solo al estudiante como tal en su vida personal y en sus relaciones interpersonales, ya que estas pueden verse influidas por esas acciones y llevar a la pérdida de amistades, $\mathrm{o}$ a aliarse con otros estudiantes que realicen acciones similares a las suyas, y con ello continuar y reforzar sus conductas conflictivas. También estas consecuencias pueden afectar la institución educativa, por la diversidad de situaciones que se deben modificar para hacer frente al conflicto, y las alteraciones a nivel de la ejecución del currículo educativo, tanto para el estudiante como para el docente.

Sin embargo, las consecuencias como tales y su efectiva aplicación, van a depender directamente de las estrategias de gestión, la capacidad, el compromiso y el profesionalismo del administrador, así como del trabajo de sus colaboradores inmediatos, ya que las implicaciones pueden ser negativas o no, no en función del conflicto sucedido, sino del abordaje que a este se le dé. Al respecto, Banz (2008), citado por López (2012), considera que "muy frecuentemente, las consecuencias negativas del conflicto no provienen del conflicto en sí mismo, sino de la manera como el conflicto es percibido y manejado por las partes" (p. 1).

De modo que las consecuencias que se desarrollen a partir de un determinado conflicto entre estudiantes ocurrido, va a depender del desenlace de estas, si van a ser negativas o positivas en función del estudiante y la propia institución educativa. Esto, en la medida que la institución pueda hacer un adecuado uso y emplear la herramienta de consecuencias; esta medida no precisamente es de represalia, sino como un posible insumo para que el estudiante pueda aprender de los sucedido. De ahí, las posibles implicaciones que esas consecuencias pueden desarrollarse a nivel estudiantil y del propio centro educativo.

\section{Metodología}

El presente estudio se aborda desde un enfoque cualitativo, empleando estrategias y técnicas para la recolección de la información como el cuestionario, el grupo focal y la entrevista. 
El diseño de esta investigación es de tipo fenomenológica, tomando en cuenta que este método va a permitir estudiar el fenómeno de los conflictos estudiantiles desde la propia óptica y vivencias de los sujetos en estudio, lo que va a favorecer ese acercamiento con la realidad existente, buscando su significación desde la intención de quienes lo viven en el centro educativo.

Para la selección de la población del estudio, se consideró el conocimiento y vivencia de la realidad en estudio en el centro educativo; por esta razón, se seleccionaron cuatro tipos de sujetos de investigación, relacionados con la temática pero desdeópticas diferentes: estudiantes, personal docente guía, profesionales en orientación y el administrador de la educación.

Por lo que, para acceder a la información de los diferentes participantes se realizó la siguiente distribución de técnicas e instrumentos: para la población estudiantil, se empleó el grupo focal, conformado por cinco estudiantes de cada nivel; para las cinco orientadoras y el director de la institución, se utilizó la técnica de entrevista semiestructurada; en el caso de los docentes guías, al ser treinta y nueve docentes, se empleó la técnica de cuestionario abierto, que era lo más pertinente al caso.

A los participantes en el estudio, se les consultó en función de las siguientes categorías de análisis:

\section{Tabla 1}

Categorías de análisis e indicadores a desarrollar en la investigación

\begin{tabular}{|c|c|}
\hline Categoría de análisis & Indicadores \\
\hline Conflictos estudiantiles & $\begin{array}{l}\text { - Desacuerdos } \\
\text { - Enfrentamientos } \\
\text { - Descontento } \\
\text { - Discusiones } \\
\text { - Discriminación } \\
\text { - Contraposición de intereses }\end{array}$ \\
\hline Categoría de análisis & Indicadores \\
\hline $\begin{array}{c}\text { Causas asociadas a conflictos } \\
\text { estudiantiles }\end{array}$ & $\begin{array}{l}\text { - Ausencia de espacios para la participación estudiantil } \\
\text { - Organización curricular } \\
\text { - Discrepancias entre la población estudiantil } \\
\text { - Rivalidad entre las población estudiantil } \\
\text { - Uso de apodos entre la población estudiantil }\end{array}$ \\
\hline
\end{tabular}




\begin{tabular}{cl}
\hline & - Problemas de autoestima \\
& - Dificultades familiares \\
& - Dificultades en las relaciones interpersonales \\
Consecuencias asociadas a & - Bajo rendimiento académico \\
conflictos estudiantiles & - Ausentismo \\
& - Fracaso escolar \\
& - Deserción \\
& - Imagen institucional \\
\hline Procesos en la prevención desde la & - Pricultades en la implementación del currículo escolar \\
gestión de la educación & - Programas de prevención dirigidos a los y las estudiantes \\
\hline \multirow{3}{*}{ - Programas de prevención dirigidas a las familias al personal docente del colegio } \\
\hline - Participación de la comunidad educativa en la resolución de conflictos \\
educación. & - Actividades y acciones que permitan la participación estudiantil en \\
& - Acciones dirigidas a la resolución alternativa de conflictos \\
\hline
\end{tabular}

Nota: Elaboración propia, 2014.

\section{Resultados, análisis y discusión}

\section{Tipos de conflictos}

En relación con los tipos de conflictos se evidenció que, dentro de este centro educativo, acontecen una serie de conflictos estudiantiles, los cuales no son desconocidos por el personal que labora en la institución educativa, desde el director, orientadoras y personal docente.

Lo que implica que, en este centro educativo, no se esté ajeno a que se desarrollen conflictos, como muy bien lo menciona uno de sus estudiantes:"(...) Diferencias de pensamientos e ideas; tal vez él que el piense algo que a mí no me guste. Choque de personalidades. La falta de respeto de uno hacia las otras demás personas, porque uno siempre trata de hacerse el grandote, de creerse el mejor (...)". Condición que ratifica una de las principales dificultades, a nivel de conflictos estudiantiles, que sucede en este centro educativo, en donde el estudiantado tiene sus diferencias, pero que no las han logrado manejar adecuadamente por lo que, a la larga, han desencadenado conflictos, tal como lo menciona un estudiante de octavo año: (...) los conflictos verbales a veces se dan, gritar cosas ofensivas entre dos o más (...)".

Lo anterior, concuerda con lo descrito por Jordan (2001), citado por López (2012), al referirse a conflictos entre estudiantes, ya que no siempre el conflicto está asociado a violencia: 
(...) no es legítimo asociar conflicto con violencia porque, mientras el conflicto responde a situaciones cotidianas de la vida social y escolar, en la que se dan enfrentamientos de intereses, discusión y necesidad de abordar el problema, la violencia es una de las maneras de enfrentarse a esta situación. (p. 82)

De acuerdo con esto, los conflictos estudiantiles relacionados con cuestiones verbales, como apodos, insultos, rivalidades y hasta irrespeto, son bastante frecuentes en este centro educativo, debido a diferentes relaciones interpersonales que se dan en la cotidianidad del Liceo, y dentro de estas van a surgir las diferencias de opiniones, rivalidades y desacuerdos, a ser expresados y afrontados de diversas maneras, tanto verbales como gestuales, evitando muchas veces el contacto físico.

Sin embargo, los conflictos estudiantiles muchas veces trascienden lo verbal y se van a la agresión física, en donde el joven pone en peligro su integridad física, como lo menciona este estudiante de octavo año: “(...) También física, a veces si por equis razón tienen algún conflicto al final del día, o la hora del almuerzo salen y van a la "urba" como todo el mundo la llama y se pelean (...)". Lo anterior, conlleva una complejidad aun mayor del fenómeno de los conflictos estudiantiles, ya que no solo suceden a lo interior del centro educativo, sino que como lo menciona este estudiante, trascienden al exterior, y tal vez cuestiones que se originan en el propio centro son llevadas fuera de este, a concretarse en expresiones y manifestaciones de agresión física entre las poblaciones estudiantiles.

Lo que implica un importante reto para la persona administradora de la educación, según Garbanzo y Orozco (2007) hacia el desarrollo sostenible del centro educativo desde la equidad, calidad y justicia en cada una de las dimensiones que esta disciplina le corresponde; de ahí que se deben crear y desarrollar las directrices, lineamientos y programas necesarios para afrontar los conflictos estudiantiles que acontecen diariamente y trabajar en mira de que los estudiantes aprenden a resolverlos, además que vean el conflicto como una herramienta de aprendizaje y desarrollo integral. Proyectos, que no solo deben ir de la mano de los lineamientos establecidos por el Ministerio de Educación Pública, sino que deben estar contextualizados a las necesidades y realidad propia de la institución educativa. Por ello, ha de ser una labor que involucre a toda la comunidad educativa, iniciando con el administrador educativo, hasta los propios estudiantes, docentes, administrativos y demás agentes colaboradores de centro.

\section{Causas de conflictos estudiantiles}

Las causas que generan conflictos estudiantiles se abordarán desde dos perspectivas: la primera, relacionada con aquellas causas internas, propias de cada estudiante y la segunda, con las causas externas al mismo. 
Son consideradas como causas internas generadoras de conflictos, todas aquellas condiciones y situaciones propias de la persona, lo que según Cascón (2006) se puede visualizar como la confluencia de diferencias, en cuanto a necesidades, intereses y hasta motivaciones. De modo, que aplicado a este centro educativo, entre los más sobresalientes factores internos causantes de conflictos estudiantiles se pueden citar, aspectos relacionados con la autoestima, ese concepto propio que tiene cada persona de sí mismo, del cual depende muchas veces su desarrollo personal y el comportamiento a nivel de relaciones interpersonales.

Otro de los factores internos que se resaltó en el estudio, no solo a nivel de opinión de los estudiantes, sino de las orientadoras también, fue lo relacionado con la comunicación, debido a que se argumenta que las personas jóvenes se manejan bajo débiles canales de comunicación, lo que implica que no siempre se comunique lo que se pretendía y mucho menos se haga de la forma adecuada. Una de las orientadoras se refiere al tema y argumenta: "(...) La comunicación fundamentalmente, siento que ahíes como la claveyen los adolescentes se ve mucho. Siento que se basan más en los que sienten, en lo que ellos escuchan y no en una comunicación asertiva con esa otra parte (...)". Punto abordado por Naranjo (2008), quien menciona que la comunicación es una temática muy compleja, pero que la sabiduría está en aprender a expresarse y escuchar. Al respecto, argumenta:

La persona que se comunica de una forma funcional es aquella que puede expresar con firmeza sus opiniones, pero también está dispuesta a aclarar y clarificar; comportarse con otra persona considerándola como un ser separado de ella y único; tratar los puntos de vista opuestos a los suyos como una oportunidad para aprender, y no como una amenaza o una señal de conflicto. (p. 11)

Línea muy delgada que sin duda persigue el hacer de la comunicación, esa herramienta por medio de la cual se puedan establecer lazos interpersonales armoniosos, de los que no solo se aprenda del otro, sino que se discuta la diversidad de opiniones y con ello, se construyan nuevas percepciones, aprendizajes y conocimiento. Lo que demanda todo un reto para el centro educativo y por ende, para el administrador de la educación, desde la creación de procesos y espacios desde el mismo salón de clases, que permitan el desarrollo de este tipo de habilidades sociales entre el estudiantado.

Entre los factores externos causantes de conflictos se encuentran todos aquellos elementos, condiciones y situaciones ajenas al estudiante pero que, de una manera u otra le afectan, debido a que este vive inmerso en ellas. Según Fernández (2007) estos factores se pueden dividir en agentes exógenos y agentes endógenos; en donde los primeros hacen referencia a elementos concernientes a la estructura social como la familia y el entorno, y los segundos a aspectos que ocurren a lo interno del centro educativo, como políticas instituciones y grupos de pares.

En relación con los agentes exógenos, los estudiantes, la dirección de colegio y las orientadoras insisten en que la parte familiar es un débil recurso en el centro educativo, no solo 
por su poca presencia, inclusive cuando se les llama, sino que también se visualiza muy poco acompañamiento de esta para con sus hijos e hijas desde la casa. Al respecto, un estudiante menciona:"(...) siento que se origina por la falta de comunicación y de contacto de los padres con sus hijos, como siempre tienen que trabajar, dejan a sus hijos solos; pasan todo el día en el colegio, llegan hasta la noche; un desinterés de parte del hogar, verdad porque no hay acompañamiento (...)".

Lo anterior implica que una débil e inadecuada influencia familiar, se configura un factor de peso no solo en el comportamiento negativo de sus hijos e hijas, sino incluso en el propio rendimiento académico de estos, ya que la familia debe ser ese sostén llamado a propiciar una formación y educación determinada. Al respecto, Martínez-Otero (2009) menciona:

(...) el clima familiar que puede ayudar a optimizar la educación y consiguientemente a neutralizar el fracaso escolar es el precedido por el afecto, la comunicación, la seguridad, el respeto, las normas, la participación y el fomento de la autonomía. (p. 81)

Se ratifica entonces, la importancia de la familia como factor protector estudiantil en tanto se asuma una posición de participación e involucramiento en la vida de las personas jóvenes, ya que lo contrario lo convierte en un factor de riesgo, causante de desequilibrio, descontrol y apatía hacia lo educativo.

Lo que sin duda, es una fuerte necesidad que desde el centro educativo se debe de atacar, para cada día atraer más a las familias e involucrarlas en los procesos educativos de sus hijos e hijas y con ello, cumplir a cabalidad con esa función formadora que, desde lo interno de cada familia, se ha de implementar.

Por otro lado, según la opinión estudiantil, los agentes endógenos más visibles en este estudio se relacionan con la imagen del colegio, ya que ha adquirido una mala reputación en los últimos años. Uno de los estudiantes menciona al respecto: "(...) gente de todo lado que ya sabe que el Liceo de Paraíso es un lugar en donde van la gente así, que anda agarrándose en todo lado, y en cualquier lado anda haciendo alboroto (...)". Lo anterior, es lamentable en la medida de que una mala imagen institucional no solo ha de repercutir negativamente en el fenómeno de los conflictos estudiantiles, debido a que el o la estudiante conflictivo podría continuar con sus inadecuadas acciones, sino que puede influir a nivel de desmotivación estudiantil de los actuales alumnos y hasta de los futuros estudiantes.

Opinión que evidencia el importante papel que adquieren aspectos como la imagen institucional para la población estudiantil y es, desde este punto que la dirección de este centro educativo debe enmarcarse para desarrollar acciones que contrarresten ese visión actual, con el fin de dar paso a estrategias para sobrellevar esta problemática y llevar al centro educativo a un futuro más sano, armonioso y llamativo que implicará ser más atractivo para las futuras generaciones de estudiantes. 
De ahí, que según Vargas (2008), el desafío más complejo de la administración de la educación es concebir y propiciar la creación de un nuevo modo de conducir el funcionamiento de los sistemas educativos, donde el eje central sea el desarrollo de capacidades humanas; desde donde el centro educativo logre posicionarse como esa institución que, a pesar de las situaciones contextuales, pueda ser un factor protector para los y las estudiantes que alberga.

\section{Consecuencias personales, académicas e institucionales a partir de conflictos estudiantiles}

A nivel de consecuencias personales, según la opinión docente, los aspectos más relevantes tienen que ver con problemas de autoestima en componentes como la imagen, seguridad, así como las alteraciones a nivel conductual que muestran algunos de los y las estudiantes, en acciones de impulsividad y agresividad. De similar forma, la población estudiantil argumenta: "(...) Para mí es la imagen, y el concepto que tienen las demás personas de uno mismo. Si uno es mujer y una va ahí y se agarra ahí en la Urba: tierrosa; porque uno tiene que saber valorarse (...)". Unido a este, otro de los aspectos afectados son relaciones interpersonales, ya que debido a esa imagen o concepto de sí mismo que la propia persona se va creando y fomentando, las personas a su alrededor empiezan a verlo de esa manera, e inclusive a rechazarlo.

Panorama difícil de enfrentar para ese estudiante partícipe de conflictos estudiantiles, ya que producto de su actuar y forma de comportarse, sus relaciones interpersonales se van a ver afectadas; condiciones que podrían ser muy significativas en la vida del joven estudiante, pues según Krauskopf (2007), en la vida del adolescente, es trascendental la influencia y papel que ejerce el grupo de pares; de su presencia o ausencia puede depender no solo la formación y socialización de ese joven, sino su propia construcción personal, a nivel de herramientas de comunicación y comportamiento. De ahí, que un estudiante que sea víctima de rechazo como consecuencia de sus acciones, puede que no logre establecer relaciones con sus iguales, desarrollar ese sentimiento de pertenencia en sus relaciones interpersonales y por ende, perder su interés por ir al colegio. Situación que no puede ser ajena y desconocida por el administrador educativo, quien debe estar al tanto de lo que ocurre en el centro educativo y de las condiciones propias que, como adolescentes, enfrentan las diferentes poblaciones estudiantiles a su cargo, con el fin de posibilitar espacios educativos más acordes y pertinentes a las características de estas.

En relación con las consecuencias académicas, las más presentes tienen que ver con la disminución del rendimiento académico del estudiante, seguido inclusive del fracaso escolar de este, lo cual va en detrimento del estudiante, quien ante un posible fracaso escolar no solo podría aumentar su ausentismo, sino hasta decidirse por abandonar el centro educativo, impulsado por el desinterés y la desmotivación que, posiblemente, le acompañan. De ahí, que la intervención de la disciplina de la administración educativa, desde el abordaje a nivel de prevención de esta temática, se hace sumamente urgente para tratar de contrarrestar, en el propio centro educativo, este tipo de situaciones tan frecuentes actualmente, como lo es la deserción estudiantil, principalmente de secundaria. 
Otro tipo de consecuencias tienen que ver con la parte institucional, específicamente con la imagen institucional, ya que según señaló la población estudiantil investigada, en este centro educativo son muy frecuentes los enfrentamientos y pleitos, por lo que la imagen del colegio se ha visto deteriorada y marcada negativamente: "(... ) La imagen del colegio es lo que afecta más, gente de todo lado que ya sabe que el Liceo de Paraíso es un lugar en donde van la gente así, que anda agarrándose en todo lado, y en cualquier lado anda haciendo alboroto (...)". Consideración muy delicada en el centro educativo, no solo por la reputación que se esté formando, sino por las consecuencias de esa caracterización a nivel de desmotivación estudiantil de sus actuales y futuros estudiantes, lo que demanda el liderazgo de la administración de la educación en la gestión de acciones para abordar el fenómeno de los conflictos estudiantiles de forma más oportuna y rigurosa, para contribuir en la creación de un clima más armonizado entre los y las estudiantes de la institución.

De modo que, según Fernández (2007), agentes como la familia, comunidad y hasta la propia institución educativa tienen la responsabilidad de que el estudiantado se desarrolle adecuadamente en su entorno; cuestión en donde radica la importancia de que estos entes se conviertan en agentes protectores y no en agentes de riesgo. De ahí, la importante labor que desde la administración de la educación se tiene, para llevar por un adecuado camino los fines y objetivos del centro educativo en donde se labore, el desarrollo de procesos y acciones para abordar esta temática de la manera más eficaz, evitando dañar o perjudicar al estudiante.

\section{Procesos que se desarrollan en la prevención de conflictos estudiantiles}

En relación con los procesos de prevención dirigidos a estudiantes y docentes guías, se hace mención a diferentes programas, entre los más señalados están los que gestiona el Departamento de Orientación y los docentes guías del centro educativo, aunque si bien no detallan a qué tipo de programas corresponden, si señalan que desde estos entes se trabaja en materia de prevención de esta temática, principalmente en los espacios de la lección semanal de orientación y guía que cada profesional tiene con sus grupos.

Al respecto, uno de los estudiantes menciona: "(...) si, las orientadoras y las guías hablan un poco de cómo solucionar los conflictos se si llegara a un punto extremo; también actividades dentro del grupo guía, ver una película o algo así (...)". Lo que resalta el papel protagónico que, en materia de prevención de conflictos estudiantiles, tiene el profesional en orientación y los propios docentes guías; es realmente valioso que, como parte de sus funciones en el centro educativo, estas personas realicen acciones para su prevención.

Sin embargo, esas acciones preventivas que lleva a cabo el centro educativo son visualizadas como insuficientes por algunos estudiantes, ya que consideran que se podrían hacer otros esfuerzos y estrategias al respecto; uno de los estudiantes menciona: “(...) No, es suficiente incluso

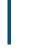


en el otro colegio que yo estaba, daban charlas y en todos los pabellones había como frases para motivar a los alumnos, que nada de bullying y todas esas cosas (...)". Lo que implica que aún existen grandes espacios vacíos en el centro educativo, por lo que deben cubrirse desde la dirección del administrador de la educación, mediante el desarrollo de programas de prevención innovadores, concisos e institucionales que, más allá de la labor del docente guía y las orientadoras, involucren a toda la comunidad educativa en acciones más micro y macro generadoras.

Además implica, según Mora (2011), que: “(...) los sistemas educativos deben estar en constante evolución, pues su propósito fundamental es el de adaptarse a las necesidades económicas, sociales, culturales y científicas del mundo actual (...)" (p. 3). De modo, que las instituciones educativas deben ir de la mano de las transformaciones y crecientes necesidades de las poblaciones estudiantiles que se atienden, no solo en materia de prevención ante los múltiples fenómenos que día a día surgen y pueden afectar al estudiante, como en el caso de conflictos estudiantiles, sino también esos eventos estudiantiles que cotidianamente acontecen en los centros educativos, a los cuales hay que darles una atención inmediata y apropiada.

En relación con los procesos de prevención, dirigidos al personal docente del centro educativo, se presenta una notoria insuficiencia en capacitación y formación en esta materia de conflictos estudiantiles, ya que según la opinión de una de las orientadores, esta se ha limitado solamente al trabajo con los protocolos del Ministerio de Educación Pública: "(...)Con ellos se trabajó los protocolos también, entonces por ahí intentamos que ellos vean que no nos podemos hacer de la vista gorda ante situaciones y problemas, no solamente de conflictos, sino que también a nivel familiar, a nivel social y todo esto (...)".

Panorama muy pobre en materia de formación y capacitación a docentes, lo que evidencia la ausencia de programas en esta línea que, necesariamente, demanda que la administración educativa de este centro educativo se tomen las medidas pertinentes para innovar y desarrollar programas de capacitación a docentes.

Desde esta visión, todo profesional en administración de la educación ha de implementar un plan de acción para la prevención e intervención de los diferentes conflictos que se presenten en la institución, como parte del proyecto organizacional y de su plan operativo institucional. (Chacón, 2013, p. 5)

Loanterior, implicaqueesos planes deacción institucional han decomprender, necesariamente, el gestionar acciones dirigidas a las poblaciones estudiantiles, así como la capacitación a sus funcionarios en diversas ramas sobre la prevención y abordaje de conflictos estudiantiles.

Otra área importante de atender corresponde al nivel familiar, espacio en el que las acciones institucionales, como la ejecución de talleres dirigidos a las familias, ocupan un lugar principal, manifiesto no solo por la opinión del personal docente sino que guarda similitud con la percepción de algunas de las orientadoras y el director de este centro educativo. El director 
argumenta: "(...) las orientadoras han venido trabajando una serie de talleres para padres, pero el primer factor es que nosotros mandamos a invitar 150 papas a un taller y llegaron $8(. .$.$) ".$

Lo que permite visualizar los esfuerzos y trabajo que se hace desde el centro educativo en función de la familia pero, como bien lo dice el director, el problema fundamental al que se han enfrentado es a la falta de compromiso y responsabilidad de padres y madres de familia, quienes tienen la costumbre de no asistir a esos espacios de formación e información que el colegio les facilita. Por ende, desde la administración de la educación se han de enriquecer ese tipo de acciones y gestionar otro tipo de estrategias innovadoras que motiven el interés de las familias para participar de los procesos de formación y educación que el centro educativo les facilita.

(...) Otro factor por considerar en el nivel administrativo de la educación, es la familia, no solo porque se establece como una célula fundamental del núcleo social, sino también porque se constituye en un factor que puede favorecer conductas de índole conflictiva y violenta en los jóvenes, ya que es la principal fuente de modelización de los niños y jóvenes, dentro de ella es donde se asimilan los valores, costumbres y hábitos que van a ser importantes a lo largo de toda su vida. (Chacón, 2013, p. 6)

De esta manera, se plantea otro reto para la administración de la educación, en tanto que la familia es un pilar fundamental en los procesos educativos y de formación que reciben los y las estudiantes en casa y que, de una u otra manera, vienen a reproducir al colegio. De ahí, que se ha de recurrir a todo tipo de esfuerzos y recursos posibles para atraer a la familia al centro educativo y ofrecerle interesantes proyectos de formación y capacitación constante, para construir y consolidar en ellos, ese factor protector en la educación de sus hijos e hijas.

De modo, que a pesar de los esfuerzos que se realizan desde la administración de la educación de este centro educativo, son evidentes los vacíos aún existentes en materia de prevención, tanto a nivel estudiantil propiamente, docentes y familiar, lo que desprende ese importante reto pendiente, a nivel organizacional, de prepararse para la prevención de conflictos estudiantiles.

\section{Procesos que se desarrollan para el abordaje de conflictos estudiantiles}

Es importante entender que del abordaje que se le dé a los conflictos estudiantiles en la institución educativa puede depender, en alguna medida, que la incidencia de estos aumente $o$ disminuya, ya que los y las estudiantes van a medir ese tipo de acciones, a fin valorar y educarse a la hora de comportarse y relacionarse con el resto de la comunidad educativa.

Considerándose este planteamiento, los conflictos estudiantiles son una de las situaciones que enfrenta continuamente la población estudiantil en los centros educativos; razón por la cual el Ministerio de Educación Pública, como garante de la educación en el país, ha emitido una serie de documentación al respecto, como el Reglamento de Evaluación de los Aprendizajes y más 
recientemente, en el 2012, emitió una serie de protocolos para atender diferentes dificultades de esta índole y otro tipo de problemáticas a nivel estudiantil.

Particularmente, en la realidad del Liceo en estudio, el personal docente guía afirma, en su mayoría, que estos protocolos han sido un buen insumo en materia de abordaje de diferentes situaciones conflictivas que ocurren entre los estudiantes y que, junto con el Reglamento de Evaluación de los Aprendizajes, son las principales herramientas que el Ministerio de Educación Pública les ha facilitado para enfrentarse a este tipo de situaciones y aplicar las normas, directrices y acciones a aquellos estudiantes que incurran en algún tipo de infracción, según las que esta documentación contempla. Lo que concuerda con la perspectiva del director, quien menciona que:"(...) ellos emitieron una serie de protocolo. Se dio una inducción a los directores de un día completo para que los trabajaran, a las orientadoras y bájenlo a los docentes, para que vallan aprendiendo del ensayo y el error (...)".

Según lo considerado anteriormente, los procesos a seguir por la institución educativa, en caso de conflictos entre estudiantes, ya se encuentran tabulados en documentación proveniente del Ministerio de Educación Pública, en alguna medida, por lo que es preciso que quien funciona como administrador educativo del centro educativo, establezca y coordine estrategias para que esas directrices realmente se pongan en marcha y se apliquen como deben ser en la institución.

(...) Hoy día las organizaciones educativas deben manejarse dentro de una concepción futurista, propositiva, flexible y responsable, donde la capacidad de adaptarse a las exigencias contextuales con innovadoras propuestas de gestión sean la característica que haga la diferencia en las organizaciones educativas de la postmodernidad; (Garbanzo y Orozco, 2010, pp. 25-27)

Lo que conlleva todo un reto para el administrador educativo, quien desde su accionar, debe gestionar aquellas acciones y lineamientos que más le convenga al centro educativo que lidera, para llevar las normativas emanadas por el Ministerio de Educación Pública a la propia realidad institucional, en lo que se involucre al personal docente y se motive la colaboración de las comunidad educativa, en general.

En relación con los procesos de abordaje dirigidos a la población estudiantil, el personal docente guía señaló, en su mayoría, que cuando ocurre algún tipo de conflicto entre estudiantes se aborda empleando recursos como el Reglamento de Evaluación de los Aprendizaje y los protocolos del Ministerio de Educación Pública; en este último caso se usa el protocolo específico para la situación que ocurra. Lo que equivale, según el Ministerio de Educación Pública, en el protocolo integral para atención de situación de violencia en los centros educativos de secundaria, a la realización de una intervención ante un conflicto de manera efectiva, desde una atención oportuna, pertinente del caso y sobre todo, orientada bajo los principios técnicos (MEP, 2012).

Como bien se menciona, cada uno de estos protocolo está diseñado para atender un fin determinado, sea la portación de armas, el matonismo u otra problemática; es a partir de 
ello, que cada protocolo señala y especifica las acciones que como centro educativo se deben ejecutar, no solo para atender al estudiante que se vea implicado en alguna de esas acciones, sino para contactar a las autoridades pertinentes, tanto a nivel interno como externo de la institución, sean padres de familia o entes de la fuerza pública, cruz roja, entre otras. De ahí que, en este caso, esta institución educativa considere el uso de cada protocolo y, específicamente el que se emplea para casos de conflictos estudiantes, como una herramienta esencial en los procesos que se presentan.

Sin embargo, el estudiantado del Liceo no percibe que las acciones que se realizan en materia de abordaje de conflictos estudiantiles en el centro educativo, sean del todo reales y efectivas, puesto que según sus criterios, en algunas ocasiones, no se observa que realmente se apliquen los artículos y sanciones pertinentes a cada caso. Uno de los estudiantes de noveno menciona: "(...) Yo he visto que tal vez se agarran y hasta con el mismo uniforme y hasta los ven y todo, y no les hacen nada; pero otras veces si se ha visto que los expulsan por los menos varios días ()". De manera que, si la existencia de los medios y recursos para aplicar sanciones se tienen a mano, pero no se sigue el proceso o no se aplican esas acciones con regularidad, es posible que los estudiantes perciban esa falta de seriedad y autoridad para la aplicación de los mismos.

De ahí, que como profesionales administradores de la educación se debe supervisar este tipo de acciones y velar para que las personas a quienes les corresponde las lleven a cabo, con el fin de lograr una gestión pertinente a esa área de acción. Al respecto, Chiavenato (2006) señala que la administración debe integrar los recursos humanos y materiales, y dirigirlos en función del alcance de los objetivos organizacionales. Así mismo, López (2012) resalta que las actitudes y acciones que se ejecuten desde la administración determinan, en gran medida, el éxito o el fracaso de los procesos que se realizan para la resolución de los conflictos.

Por ello, el administrador educativo debe procurar el buen funcionamiento del recurso humano que tenga a su disposición para que, en materia de abordar las situaciones conflictivas, se les posibilite a los y las estudiantes la atención que se requiere en cada caso, el empleo de las acciones pertinentes ejercidas por las personas que les corresponde a nivel institucional, pues de eso dependerá el fracaso o éxito del abordaje de los mismos.

Los actores educativos que este estudio ha permitido visualizar de forma protagónica en el abordaje de conflictos estudiantiles, son los docentes guías y profesionales en orientación; en tanto, una de las orientadoras menciona: "() Cuando ocurre una falta tienen que llevar el debido proceso, entonces el profesor guía tiene que citar a los padres de familia, citar a las personas relacionadas con el conflicto, tomar declaraciones, esto en compañía con el orientador (...)", asunto que el mismo Reglamento de Evaluación de los Aprendizajes, en su artículo 86, determina que son los docente guías, con ayuda del profesional en orientación, los encargados de llevar los debidos procesos disciplinarios en los estudiantes. Así mismo, este Reglamento, en su artículo 73, menciona que: "las faltas en las que incurran los estudiantes tendrán consecuencias en el 
proceso de definición de la nota de conducta de cada periodo " (MEP, 2009, p. 57). De modo que, cuando un estudiante incurre en algún tipo de falta como lo son los conflictos entre estudiantes, uno de los aspectos que se verá afectado es su nota de conducta.

De similar manera, la población estudiantil considera que ambas figuras, docente guía y orientadoras, son los profesionales que normalmente intervienen cuando se deben abordar situaciones conflictivas entre estudiantes. Al respeto, un estudiante argumenta: "(...) Siempre llaman a las orientadoras, cuando pasa algún conflicto. El profeguía también llama a los estudiantes para hablar con ellos (...)".

Sin embargo, este tipo de acciones, más a nivel institucional para el abordaje de los conflictos, han de implicar una gestión eficaz del administrador educativo, quien desde su compromiso y colaboración, no se puede quedar solo con la labor del docente guía y profesional en orientación, sino que debe involucrar a los demás colaboradores del centro educativo, en la práctica de procesos para abordar y atender situaciones conflictivas.

La gestión de los conflictos consiste en un tratamiento pedagógico, cuya piedra angular se constituye en la mediación pedagógica y la instrumentalización de diversas estrategias que colaboren con una toma de conciencia y puesta en práctica de valores y principios fundamentales para el fomento de una sana convivencia ( ). (Chacón, 2013, p. 12)

De modo, que es necesario que el administrador de la educación de este centro educativo, se convierta en ese gestor y mediador que, desde su investidura y liderazgo, sea precursor de transformaciones y posibilite la realización de diferentes actividades, acciones y procesos, con el objetivo de buscar un mejoramiento continuo de la organización que lidera. Desde las cuales, involucre a la comunidad educativa, no solo a nivel de denuncia de situaciones conflictivas entre estudiantes, sino que cada quien, sea el mismo docente guía, profesionales en orientación y demás personal, asuma el rol que debe y se apliquen los procesos de atención y disciplina al estudiante, pertinentes para cada caso.

Al preguntarse sobre la efectividad o no de esos procesos de abordaje, las opiniones están un poco dividas entre los diferentes sujetos investigados. En el caso del personal docente guía, argumenta que existen razones para pensar que las acciones si han sido efectivas, pero que también hay razones para expresar lo contrario, aunque se inclinan más en la posición de que si han mejorado, ya que desde una adecuada aplicación de los debidos procesos disciplinarios y el compromiso de las personas encargadas de ejecutarlas, el cima institucional ha mejorado, por lo que el índice de conflictos, principalmente aquellos relacionados con violencia física, han disminuido considerablemente.

No obstante, desde la opinión de algunos estudiantes, el panorama es otro y, al parecer, las acciones no han sido tan efectivas; uno de los estudiantes argumenta: "(...) Por ejemplo hubo un pleito hace poco, ahí por las aulas de diseño, que el güila era conocido mí y nada más me dijeron, lo expulsaron por no sé cuánto, era por un mes casi, y cuando me di cuenta no eran ni 15 días 
y ya está aquí otra vez (...)". Donde impera un total desconocimiento, por parte de la población estudiantil, en materia de si realmente se aplican las acciones pertinentes y hasta sanciones a los estudiantes que ven implicados en conflictos estudiantiles.

Pero no solo la falta de información es evidente en los estudiantes, sino que ellos también afirman que hace falta mano dura por parte de las autoridades del colegio para que las acciones, en materia de sanciones ante el abordaje de conflictos, realmente se cumplan y así, mejorar la convivencia. En consideración, uno de los estudiantes menciona: "(...) Pienso que aquí como la Dirección y las orientadoras, la autoridad no la tienen, porque si de verdad quieren tener un colegio bien, que ya no esté manchado, que no estén hablando de él, tienen que actuar, como te digo, expulsar a las personas causantes de problemas (...)".

Desde esta posición, para una gestión de los conflictos más óptima, no solo es necesario un accionar de calidad por parte del administrador de educación del centro educativo, sino que es preciso involucrar y comprometer a otros actores educativos. Como lo menciona Chacón (2013), gestionar conflictos significa que quien funcione como profesional de la administración en un centro educativo se constituya en ese líder transformacional de su organización, de modo que sea un agente de cambio que impulse en la comunidad educativa valores como la convivencia, la solidaridad, el respeto y la participación. Lo que implica, que se motive al personal a cargo a que cumpla los debidos procesos para que, realmente abarquen las acciones necesarias y que, al alumnado se le muestre, con seriedad y compromiso, que cada acción que se hace es en miras de lograr mejorar la convivencia estudiantil y el clima institucional.

\section{Conclusiones}

A partir de los hallazgos manifestados anteriormente como producto de esta investigación, se evidencia que, dentro de este centro educativo, acontecen una serie de conflictos estudiantiles verbales que van desde insultos, apodos, bromas y hasta desacuerdos, rivalidades y discusiones. Muchos de ellos, son producto de problemas de comunicación y están asociados a aspectos de ausencia de valores para una sana convivencia, como el respeto y la tolerancia.

En relación con los conflictos físicos, es notoria su presencia en acciones entre estudiantes, como pleitos y golpes, tanto fuera como dentro del centro educativo, pero no en igual grado que las verbales.

En el caso de las causas internas generadoras de conflictos, las más señaladas giran alrededor de problemas estudiantiles relacionados con la autoestima y dificultades para comunicarse adecuadamente con sus iguales, así como su etapa del desarrollo, la adolescencia, y todos los cambios que en este periodo se experimentan. Por lo que se hace necesaria una gestión que favorezca una educación más integral en el centro educativo, para contrarrestar esta situación. 
En relación con las causas externas, las más preponderantes se relacionan con las dificultades a nivel familiar que experimenta la población estudiantil, concernientes al escaso acompañamiento de las familias y las dificultades en el clima familiar en que se encuentran inmersos. Lo que es de total conocimiento de las autoridades del centro educativo, por lo que se requiere implementar nuevas estrategias y medios, desde la administración de la educación de este centro educativo, para involucrar a la familia en acciones propias de la institución y con ello, en la educación de sus hijos e hijas.

En cuanto a las consecuencias para cada estudiante que participa de conflictos, se evidencian problemas de autoestima, ejemplificados en la desvaloración que experimenta el estudiante, las dificultades al entablar relaciones interpersonales, así como la disminución del rendimiento académico y el ausentismo. De ahí, que desde la gestión de la educación se hace necesario conocer más aspectos como la epata de desarrollo, competencias y limitaciones de las poblaciones estudiantiles que se atienden, a fin de facilitar una atención, desde el centro educativo, según la necesidades y aspiraciones de los mismos, para evitar, en alguna medida, aspectos como el ausentismo y hasta la deserción del estudiante del sistema educativo.

En las consecuencias institucionales, es importante señalar la imagen institucional, ya que se rescata que en este caso se ha deteriorado en los últimos años, debido a la gran cantidad de eventos conflictivos, tanto verbales como físicos, que protagonizan sus estudiantes. Lo que es muy delicado y requiere especial atención desde la administración educativa de este centro educativo, ya que del cambio de esa imagen hacia un conceptualización más positiva puede depender el futuro de la institución a nivel de motivación estudiantil y para efectos de matrícula estudiantil a futuro.

En el caso de los programas de prevención de conflictos estudiantiles dirigidos a la población estudiantil, se concluye que no existen como tales; sin embargo, si se realizan algunas acciones al respecto y los únicos que abordan el tema, en alguna medida, son el Departamento de Orientación, en primer lugar y en segundo lugar, el docente guía. Situación que amerita un cambio en la percepción institucional, ya que esta debe ser una labor más colaborativa que involucre a toda la comunidad educativa, bajo la dirección del administrador de la educación.

Los programas de prevención de conflictos estudiantiles dirigidos al personal, tampoco existen, aunque si se realizan algunas acciones que se limitan a que se les informe para la aplicación de los protocolos del Ministerio de Educación Pública, así como del Reglamento de Evaluación de los Aprendizajes. Reflejándose un fuerte vacío que se ejerce desde la administración de ese centro educativo, ya que se hace necesario favorecer nuevos procesos de formación constante dirigidos a personal docente.

En el caso de los programas de prevención de conflictos estudiantiles dirigidos a la familia, parece ser que son muy pocos los que se ejecutan, pero si se hacen esfuerzos desde el centro educativo por realizar acciones como talleres con padres y madres de familia, los cuales presentan el obstáculo de la inasistencia familiar. 
En materia de abordaje de conflictos en este centro educativo, los principales encargados son los docentes guías y las orientadoras, quienes en su mayoría son los que ejecutan los procesos de mediación, aplicación de protocolos del Ministerio de Educación Pública y los debidos procesos disciplinarios, descritos en el Reglamento de Evaluación de los Aprendizajes.

En cuanto a la efectividad o no de los procesos de abordaje de los conflictos estudiantiles, la población estudiantil considerada, en su mayoría, opina que no son efectivos, debido a la falta de autoridad y rigurosidad que se emplea desde la administración del colegio. De ahí, la necesidad de un abordaje más riguroso y adecuada aplicación de normas establecidas a la hora de manejarse un conflicto.

Desde la administración de la educación existe cierto tipo de debilidades a nivel del tema de prevención y abordaje de conflictos estudiantiles, ya queal parecer no existen programas o proyectos, a nivel institucional, bien definidos para tratar estos elementos. Lo que implica un importante reto para la administración educativa de este centro educativo, quien debe innovar y gestionar procesos en procura de satisfacer esta necesidad y que requiere, necesariamente, atención inmediata.

\section{Referencias bibliográficas}

Arroyo, J. A. (2009). Gestión directiva del currículo. Revista Actualidades Investigativas en Educación, 9 (2), 1-17. Recuperado en: http://revista.inie.ucr.ac.cr/ediciones/controlador/ Article/accion/show/articulo/gestio n-directiva-del-curriculum.htm

Botero, C. (2008). Cinco tendencias de la gestión educativa. Revista hispanoamericana de Educación, 49 (2). Recuperado en: http://www.rieoei.org/deloslectores/2811Botero.pdf

Cascón, F. (2006). Apuntes sobre educar en y para el conflicto y la convivencia. Revista En portada, 55. Recuperado en: http://pacoc.pangea.org/documentos/_andalucia_educativa_paco.pdf

Chacón, A. (2012). La gestión de los conflictos estudiantiles: un enfoque desde la administración de la educación. Revista Gestión de la Educación, 2(1). Recuperado en: file:///C:/Users/admin/ Downloads/8510-12058-1-SM.pdf

Chacón, A. (2013). Propuesta para la elaboración de un manual para la gestión de los conflictos estudiantiles. Revista Gestión de la Educación, 4 (1), 1-35. Recuperado en: file:///C:/Users/ admin/Downloads/12918-21325-3-PB.pdf 
Chiavenato, I. (2006) Introducción a la teoría general de la administración. Colombia: McGraw Hill Interamericana.

Dengo, M. E. (2011). Educación costarricense. San José, Costa Rica: EUNED.

Fernández, I. (2007). Prevención de la violencia y resolución de conflictos. Madrid: Narcea, S.A.

Garbanzo, G. M. y Orozco, V. H. (2007). Desafíos del sistema educativo costarricense: un nuevo paradigmadelaadministración delaeducación. RevistaEducación,31(2),95-110. Recuperado en: http://www.revistas.ucr.ac.cr/index.php/educacion/article/viewFile/1246/1309

Garbanzo, G. M. y Orozco, V. H. (2010). Liderazgo para una gestión moderna de procesos educativo. Revista Educación, 34(1), 15-29. Recuperado en: http://www.redalyc.org/ articulo.oa?id=44013961001

Krauskopf, D. (2007). Adolescencia y educación. San José, Costa Rica: EUNED.

López, L. (2012). Abordaje de los conflictos estudiantiles en la gestión de las Escuelas Barrio Limoncito y la Colina, del circuito escolar 02, de la Dirección Regional de Educación de Limón. (Tesis de Maestría, Universidad de Costa Rica). Sede del Caribe, Limón, Costa Rica.

López, L. (2012). Abordaje de los conflictos estudiantiles en la gestión de las organizaciones educativas de primaria. Revista Gestión de la Educación, 2 (2), 1-33. Recuperado en: file:///C:/ Users/admin/Downloads/5869-12252-1-PB.pdf

Martínez-Otero P., V. (2009). Diversos condicionantes del fracaso escolar en la educación secundaria. Revista Iberoamericana de Educación, 51, 67-85. Recuperado en: http://www. rieoei.org/rie51a03.htm

Ministerio de Educación Pública. (2009). Reglamento de Evaluación de los Aprendizajes. Recuperado en: http://www.apse.or.cr/webapse/legdoc/leg03.htm 
Ministerio de Educación Pública. (2012). Protocolo integrado para la atención de situaciones de violencia en los centros educativos de secundaria. Recuperado en: http://www.mep.go.cr/ sites/default/files/page/adjuntos/protocolointegrado.pdf

Mora, C. (2011). Labor del Coordinador Académico en la gestión del currículo en las organizaciones educativas de secundaria pública. Revista Gestión de la Educación, 1 (2), 1-34. Recuperado en: file:///C:/Users/admin/Downloads/2142-3427-1-SM.pdf

Naranjo, M. L. (2008). Relaciones Interpersonales adecuadas mediante una comunicación y conductas asertivas. RevistaActualidades Investigativas en Educación, 8(1) ,1-27. Recuperado en: http://revista.inie.ucr.ac.cr/uploads/tx_magazine/asertiva.pdf

Rodríguez, A. (2006). Educación formativa. Recuperado en: http://www.edumedia.org.ve/ productos/Apoyo/detalle.asp?id=63

Rodríguez, J. M. (2008). Los docentes ante las situaciones de violencia escolar. Revista Electrónica Interuniversitaria de Formación del Profesorado, 27 (11). Recuperado en: http://www. redalyc.org/articulo.oa?id=217015205005

Torres, C. (2004) Educación, poder y biografía. México: Siglo XXI.

Vargas, I. (2008). Análisis de cinco desafíos en el ejercicio de la administración educativa. Revista Actualidades Investigativas en Educación, 8(1), 1-15. Recuperado en: http://revista.inie.ucr. ac.cr/autores/controlador/Article/accion/show/articulo/analisis-de-cinco-desafios-en-elejercicio-de-la-administracion-educativa.html

Viñas C., J. (2011). Conflictos en centros educativos cultura organizativa y mediación para la convivencia. Barcelona: Editorial GRAÓ. 